\title{
Comment on "Review of Experimental Studies of Secondary Ice Production" by Korolev and Leisner (2020)
}

\author{
By Vaughan T. J. Phillips ${ }^{1}$, Jun-Ichi Yano ${ }^{2}$, Akash Deshmukh ${ }^{1}$ and Deepak Waman ${ }^{1}$
}

5

\author{
${ }^{1}$ Department of Physical Geography, University of Lund, Lund, Sweden \\ ${ }^{2}$ CNRM, UMR 3589 (CNRS), Meteo-France, 31057 Toulouse Cedex, France \\ Correspondence to: Vaughan Phillips (Vaughan.phillips@nateko.lu.se)
}

Abstract. This is a comment on the article 'Review of Experimental Studies of Secondary Ice Production' by Korolev and

10 Leisner (2020, hereafter termed 'KL2020'), referring to the discussion about ice fragmentation. We argue that the only two studies characterising fragmentation in ice-ice collisions are not so erroneous as to prevent their use in representing this breakup in numerical models, contrary to the impression given in the review. A scaling analysis suggests that breakup of ice during sublimation can make a significant, albeit lesser, contribution to ice enhancement in clouds.

\section{Introduction}

15 The literature of secondary ice production was recently reviewed by KL2020. The focus of their review is on laboratory experiments. It is commendable that their review attempts to re-invigorate laboratory observations of the various types of fragmentation of ice, research that has in the last decade been oriented chiefly towards heterogeneous ice nucleation. Regarding observations of breakup in ice-ice collisions, only two prior lab studies have ever been published quantifying it (Vardiman 1978; Takahashi et al. 1995).

20

However, the unfortunate impression is given to the reader that numerical modeling and theoretical studies of breakup in iceice collisions are somehow currently impossible due to the fact that reliable data for laboratory experiments are critically missing at present. Even if the available data were unreliable, which is not the case, some stand-alone theoretical and modelling studies are nevertheless possible by developing a formulation with a framework that solely relies on theoretical studies from statistical physics. The purpose of this comment is to point out some mistakes arising in their review, as a result.

The focus here is on their Section 4, which covered this topic of fragmentation of ice by breakup in ice-ice collisions, because that is where we have made critical contributions both in theories and modelling recently. KL2020 have issued a corrigendum for how this section deals with three of our earlier studies about fragmentation of ice are cited in their corrected review (Yano 
30 and Phillips 2011; Yano et al. 2016; Phillips et al. 2017a). Before the correction, a fourth study (Phillips et al. 2018) was cited erroneously.

The next section first summarizes our theoretical and modeling contributions. That is followed by comments on the review by Korolev and Leisner (2020, their Section 4) and its corrigendum in Sec. 3. This describes how the review by KL2020 made

35 several basic mistakes in their survey of prior lab studies underpinning our published modeling of fragmentation of ice in iceice collisions. The present comment describes these errors, which their Corrigendum has not rectified.

\section{Our Theoretical and Modelling Contributions}

Yano and Phillips (2011) and Yano et al. (2016) provided a 0D analytical model of a cloud. Both studies developed a theory

40 of the nonlinear growth of ice concentrations with three species of ice: crystals, small graupel and large graupel. It was found that a sole non-dimensional parameter, $\tilde{c}$, which measures the efficiency of ice multiplication, characterizes the system in its $2 \mathrm{D}$ phase-space. A systematic investigation of the behavior of the system is performed by varying this nondimensional parameter. A tendency for explosive ice-multiplication is identified in a regime with $\tilde{c}>1$.

45 The laboratory experiment by Takahashi et al. (1995) was referred to in both papers (Yano and Phillips 2011; Yano et al. 2016) solely for the purpose of obtaining an estimate for number of ice fragments per collision $(N)$, which is a variable required for estimating the order of magnitude of the nondimensional parameter, $\tilde{c}$. The obtained estimate, $\tilde{c} \sim 10^{2}$, is far above the critical value (unity) required for explosive multiplication. In this respect, those theoretical studies do not depend on any results of laboratory experiments in any critical manner. Additionally, Yano and Phillips (2016) further considered a contribution of

50 stochastic fluctuations of the ice-fragmentation number by collision. The paper shows that multiplicative noise effect induced by this stochasticity may lead to an explosive multiplication even under a subcritical state (i.e., $\tilde{c}<1$ ).

More specifically, Figure 1 illustrates the lab experiment by Takahashi et al. (1995) how collisions between two giant spheres of ice ( $2 \mathrm{~cm}$ diameter), one rimed (A) and the other unrimed (B), were performed. The unrimed sphere was fixed while the

55 rimed one was on a rotating arm. Both particles were intended to be representative of small and large graupel colliding in a real cloud after falling into weak LWC conditions, where the smaller one grows by vapour deposition predominantly and the other by riming mostly, as observed by an airborne video-probe (Takahashi 1993; Takahashi and Kuhara 1993). Those studies in 1993 reported high concentrations of ice observed for such collisions. Takahashi et al. (1995) in the lab experiment observed hundreds of ice fragments per collision $(N)$, and provided a reduced estimate of $N$ for natural graupel of more common 60 millimeter-sizes (about 50). 


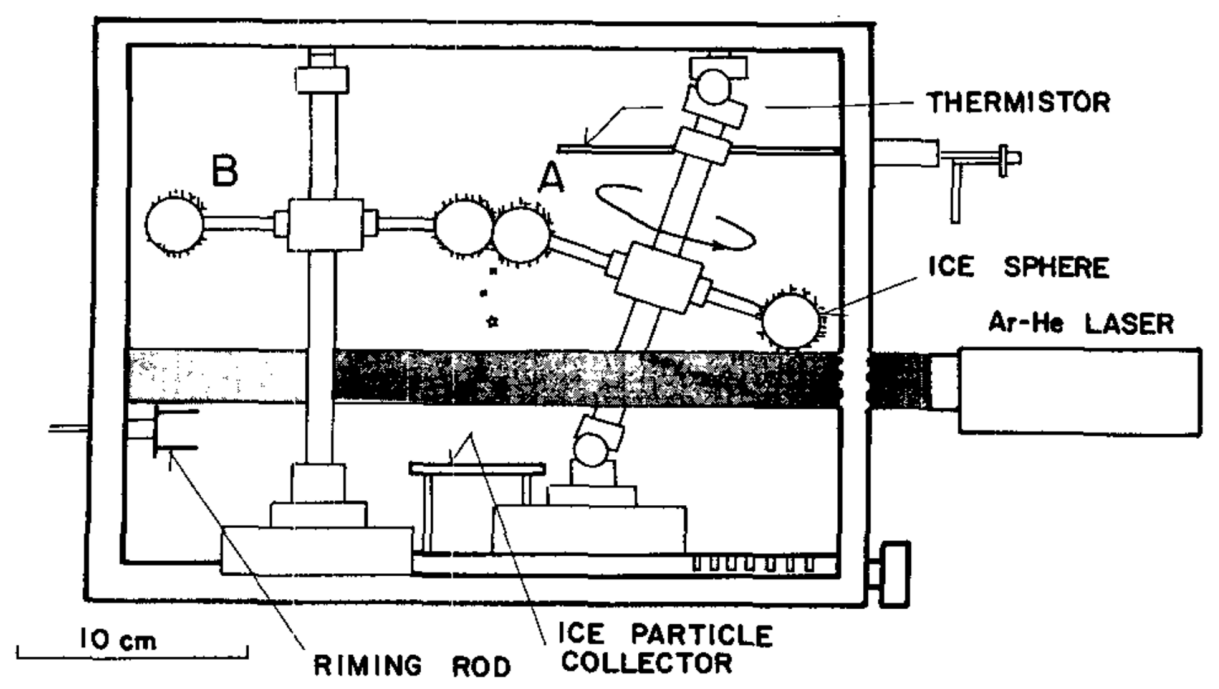

Figure 1: Experimental apparatus of Takahashi et al. (1995) to observe fragmentation in ice-ice collisions: $(A)$ ice spheres rotated at a tangential speed of $4 \mathrm{~m} \mathrm{~s}^{-1}$, while (B) ice spheres were stationary. The ejected ice particles were collected on the plate below. Cloud droplets were supplied from the centre of the right wall. From Takahashi et al. (1995). (C) American Meteorological Society. Used with permission.

Yano and Phillips (2011) and Yano et al. (2016) used that published rescaled estimate of $N$ (50 splinters) for the standard case. However, both papers did not provide any theory about the physics of breakup in a given collision at the particle scale, and rather, merely explored theoretically the overall effects on any cloud. By contrast, Phillips et al. (2017a) created a formulation to predict the number of fragments of ice emitted in any collision of two ice particles, as a function of their sizes, velocities, temperature and morphology. It was based on the robust formulations from theoretical statistical physics considering energy conservation at the particle-scale. For a pair of colliding particles that initially are not rotating:

$$
K_{0}=K_{1}+\Delta S+K_{t h}
$$

Here $K_{0}$ is the initial CKE, while $K_{1}$ is the final kinetic energy of the system after impact in the frame of reference of the center of mass, consisting principally of the CKE of the colliding particles, but also their rotational kinetic energy. This coefficient of restitution (Wall et al. 1990; Supulver et al. 1995) is not restricted to head-on collisions and includes the possibility of oblique collisions with rotation afterwards. This, together with observed statistics of surface asperities, led to the formulation: 


$$
N=\alpha A(T, D \ldots)\left(1-\exp \left[-\left(\frac{C K_{0}}{\alpha A(T, D, . .-)}\right)^{\gamma}\right]\right)
$$

Here, $N$ is the number of fragments per collision, $K_{0}$ is the initial collision kinetic energy (CKE) and $\alpha$ is surface area

80 (equivalent spherical) of the smaller particle in the colliding pair, while $A(T, D \ldots), \gamma$ and $C$ are empirical constants expressing how fragility depends on ice morphology and ambient conditions. Figure 2 shows how the general mathematical form of Eq (2) is consistent with independent data from Vidaurre and Hallett (2009) for an extreme speed (130 m/s) and a wide range of sizes.

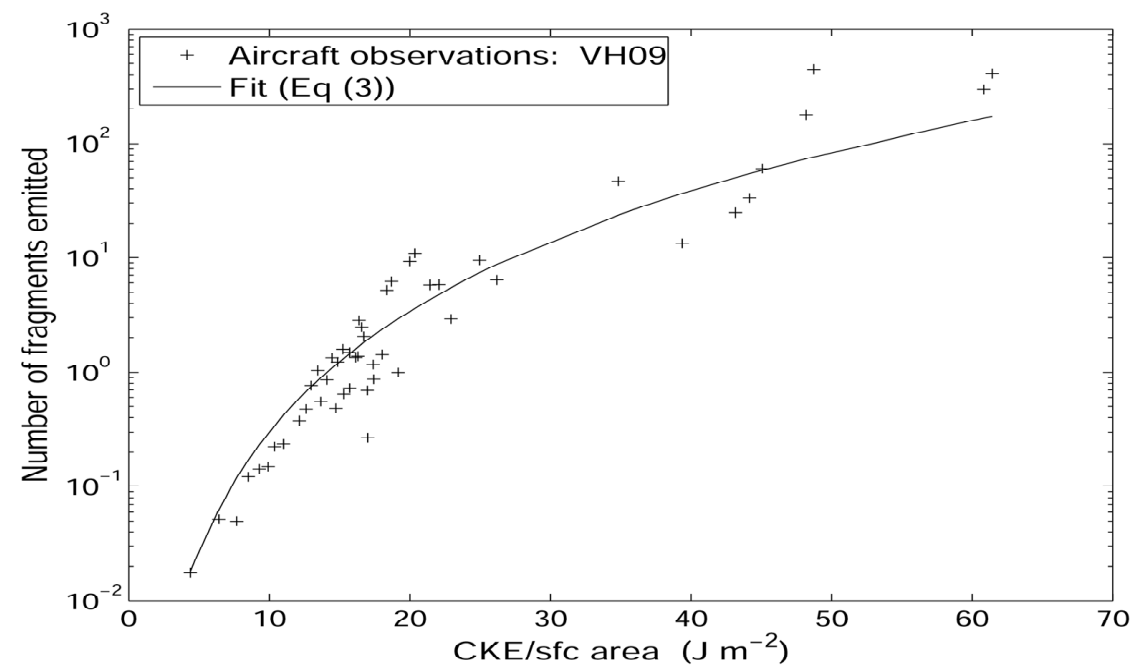

Figure 2: Measurements by Vidaurre and Hallett (2009) of numbers of fragments from ice crystals of many sizes $(10-300 \mu \mathrm{m})$ impacting the formvar replicator flown at $130 \mathrm{~ms}^{-1}$ through clouds above Oklahoma, plotted as a function of the ratio of CKE to surface area of the ice particle. From Phillips et al. (2017a).

The empirical constants of the theory (Eq (2)) were constrained for graupel-graupel collisions over a wide range of CKEs and impact speeds by the lab experiment from Takahashi et al. (1995), (Fig. 3). For other microphysical species, these constants were constrained by observations with a cloud chamber outdoors on a mountainside by Vardiman (1978), (Figure 4). Thus, splintering for each permutation of microphysical species in ice-ice collisions between snow, crystals, graupel and hail was 90 predicted in our formulation. 


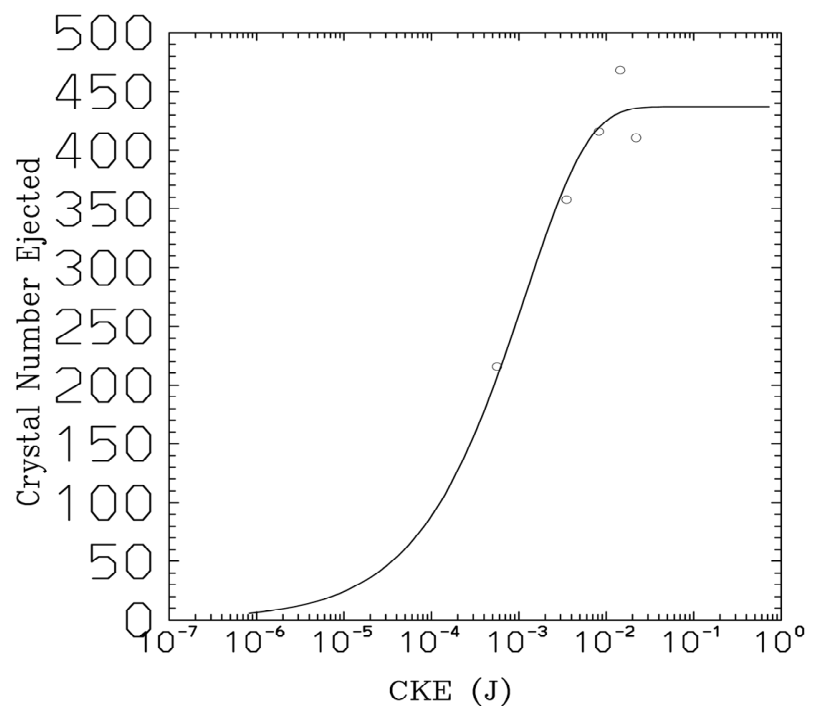

Figure 3: Measurements of numbers of fragments per hail-hail (2-cm diameter) collision (circles), as a function of initial CKE, which we inferred using Hertz theory from the published data of the experiment by Takahashi et al. (1995). From Phillips et al. (2017a).

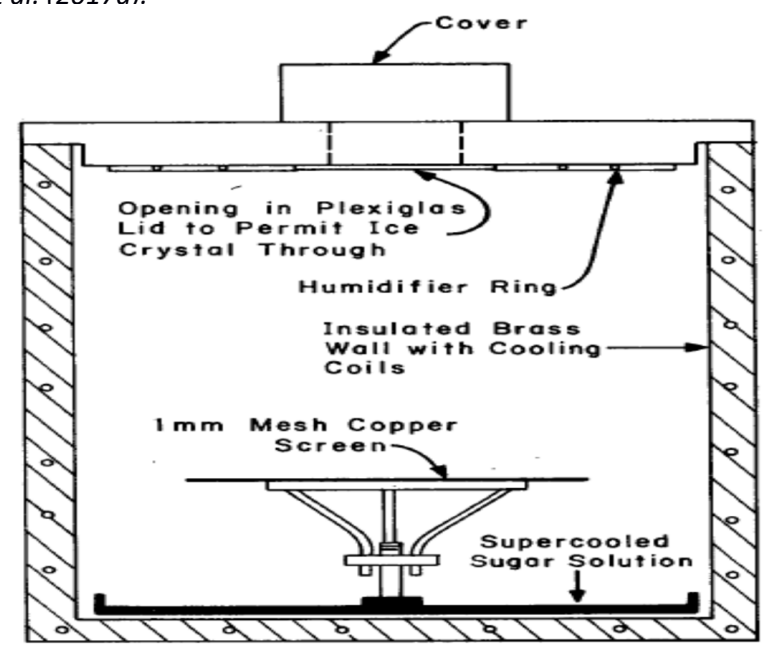

Figure 4: The portable instrument used to determine the number of fragments from natural falling ice particles. It was placed outdoors on the ground with natural ice falling through the hole in the top and into the chamber, breaking on impact with the screen. From Vardiman (1978). (C) American Meteorological Society. Used with permission. 
Phillips et al. (2017b) applied the formulation in the 'aerosol-cloud model' (AC) to quantify the role of ice-ice collisional breakup for a convective storm observed by radar and aircraft over the US High Plains (STEPS). Ice-ice breakup generated over $99 \%$ of all non-homogeneously nucleated ice particles, and was needed for agreement of ice concentrations predicted by AC with aircraft observations, which were corrected for artificial shattering biases. Only with our scheme for breakup included were various observations (e.g. supercooled liquid water content) of the clouds reproduced. This discovery was never mentioned in the review by KL2020.

Finally, by pooling published lab observations of freezing drops in free-fall, a formulation was created for secondary ice production by fragmentation of raindrops freezing quasi-spherically ('Mode 1'), (Phillips et al. 2018). By theoretical considerations, an alternative type of secondary ice production from collisions of a raindrop with a more massive ice particle was represented ('Mode 2'). The formulation for both modes was applied in a detailed bin microphysics parcel simulation and shown to reproduce aircraft observations of cloud glaciation for a tropical convective case.

\section{Comments on the Review Paper and its Corrigendum}

\subsection{Breakup in Ice-Ice Collisions}

This is what was written in the review by Korolev and Leisner (2020, their Section 4) in the section about fragmentation in ice-ice collisions, after including the corrections from the corrigendum: corrigendum deleting mention of our work]. These studies were based on the consideration of collisional kinetic energy and linear momentum. Such considerations would be relevant only for cases of direct central impact. In a general case, angular momentum and rotational energy should be taken into consideration. Since oblique particle collisions are more frequent than central collision, the efficiency of SIP obtained in these works is expected to be overestimated.

The theoretical framework of collisional fragmentation developed in Yano and Phillips (2011), Yano et al. (2016), and Phillips et al. (2017[a]) was calibrated against experimental results of Vardiman (1978) and Takahashi et al. 
(1995), [sentence A, corrected by the corrigendum]. A detailed analysis of the Takahashi et al. (1995) laboratory setup indicated that the riming of ice spheres occurred in still air, which resulted in more lumpy and fragile rime compared to that formed in free-falling graupel.

The collisional kinetic energy and the surface area of collision of the $2 \mathrm{~cm}$ diameter ice spheres also significantly exceed the kinetic energy and collision area of graupel whose typical size is a few millimeters. Altogether, it may result in overestimation of the rate of SIP, compared to graupel formed in natural clouds.

...No parameterizations of SIP due to ice-ice collisional fragmentation can be developed at that stage based on two laboratory observations, whose results are conflicting with each other. [sentence B]"

135 Sentence A would have been more accurate if it had mentioned that Yano and Phillips $(2011,2016)$ and Yano et al. (2016) provided a theory of only cloud glaciation and did not focus on fragmentation at the particle-scale. They did not use data from Vardiman (1978).

The corrigendum is hardly satisfactory with the failure by KL2020 to retract their more serious errors. Contrary to the 140 impression given by the second and third quoted paragraphs above, our formulation of fragmentation of any given ice-ice collision (Phillips et al. 2017a) is in fact not based on simple curve-fitting of laboratory/field results but rather has a robust theoretical basis (Sec. 2). The fundamental quantities determining fragment numbers per collision are not merely particle size and temperature per se, but rather are the initial CKE and contact area (Eq (1)). Equally, regarding our 0D analytical model of cloud glaciation, the theoretical results reported in Yano and Phillips (2011) and Yano et al. (2016) do not depend on

145 laboratory results in any sensitive manner; a lab observation of $N$ is merely used for an estimate a value of the non-dimensional multiplication-efficiency parameter, $c$, along with several other values. That investigation is not fixed to this estimated value, but instead is performed over the full range of possible values of this non-dimensional parameter. In short, their review continues to give a misleading picture of our work.

150 Even with the corrigendum, KL2020 still negatively criticise the experimental results of Vardiman and Takahashi et al. (1995), which provided estimates of some parameters in the theoretical formulation by Phillips et al. (2017a). In particular, their contentious claim has not been retracted about the impossibility of applying both lab/field studies for any formulation (sentence B). In fact, our overall theoretical formulation itself is developed in a general sense, as a versatile framework independent of any particular laboratory experiment. Their statement (sentence B) effectively casts doubt on the integrity of our formulation 
Their negative criticisms of both lab/field experiments (Takahashi et al. 1995; Vardiman 1978) are exaggerated, because our theory allows us to relate numbers of fragments to the fundamental determinative quantity, namely initial kinetic energy, for any collision. This allows us to quantify the errors inherent in published measurements from various simplifications in the design of both experiments. The errors, when quantified, transpire to be quite minimal in terms of numbers of ice particles emitted from any collision.

First, as noted in the above quote, KL2020 criticize Vardiman (1978) for failing to include rotational energy from oblique collisions in their theory. Korolev and Leisner (2020, personal communication) also extend this criticism to include a similar omission in actual observations by Takahashi et al. (1995) and Vardiman (1978). Yet it can be shown that the final rotational energy is only a small fraction of the initial CKE. For any sphere of size, $R$, and mass, $M$, its moment of inertia is $I=$ $(2 / 5) M R^{2}$. Consider a strongly oblique collision between two spheres of different sizes and assuming very frictional surfaces of both. During the rebounding part of the period of contact, the ratio of rotational to translational kinetic energy of the smaller sphere is approximated by that of a sphere rolling on a flat surface: $2 / 5$. At the other extreme, for a head-on collision between such spheres there is zero rotation afterwards. Thus, by this assumption of very frictional surfaces, the average fraction of initial CKE converted to rotational energy, accounting for all possible angles of collision, would be of the order of $10 \%$. In fact, this must be an upper bound (10\%), since Supulver et al. (1995) observed slow ice-ice collisions of 5-cm ice spheres in the lab and found friction is insignificant, implying little transfer to rotational energy in nature.

175 Consequently, the error in either the measured or predicted numbers of fragments per collision, introduced by artificially preventing any rotational evasive response on rebound, is minimal. That omission only weakly affects the energy available for fragmentation during impact (Eqs (1), (2)). This available energy is related to the difference between initial and final total kinetic energies. For example, a 10\% change (the upper bound noted above) in the initial CKE would correspond to only a $1 \%$ change in fragments emitted, in view of our analysis of observations by Takahashi et al. at various impact speeds (Phillips

180 et al. 2017a), (Fig. 3). Thus, the omission of rotational evasive rebound from oblique impacts in Vardiman's theory, and in either of the two lab/field experiments, cannot seriously have biased the fragmentation per collision. Additionally, by similar arguments, an estimated effect on the coefficient of restitution for ice-ice impacts (e.g. Supulver et al. 1995) arising from fixing the target can be shown to cause only a limited change in energy available for fragmentation and little change of number of fragments.

Second, it is not true that any "overestimation of the rate of SIP" must arise from the fact that " $2 \mathrm{~cm}$ diameter ice spheres also significantly exceed the kinetic energy and collision area of [most natural] graupel", as KL2020 still claim. The formulation of Phillips et al. (2017a) represents the fundamental dependence of fragmentation with both CKE and surface area of contact realistically (Sec. 2): the numbers of fragments per collision observed by Takahashi et al. (1995) for a wide range of impact speeds (or CKEs) were realistically reproduced by our theory (Phillips et al. 2017a), (Fig. 3). The fact that we fitted the theory 
to the giant spheres of the lab experiment of Takahashi et al. (1995) does not make it erroneous for smaller sizes, because our formulation (Eqs (1), (2)) has a sound theoretical basis on the conservation of energy and is universally applicable to particles of all sizes and morphologies.

195 Third, although the unrimed ice sphere in each collision observed by Takahashi et al. (1995) would be expected to have a "fragile" surface, they selected this combination of surface morphologies (rimed and unrimed, depositional and riming growth respectively) because they judged it to be representative for real in-cloud situations of ice multiplication that they had observed directly. Previously, fall-out of large graupel into regions of weak LWC with small graupel had been observed in situ by Takahashi (1993) to generate copious ice crystals — with a novel video probe. High concentrations of ice crystals co-located with graupel had been observed in the mixed-phase region of deep convective clouds with the video probe flown on a balloon by Takahashi and Kuhara (1993), providing indisputable evidence of ice morphology and size. Such measurements by balloon are without the artificial shattering biases afflicting some airborne probes.

Consequently, Takahashi et al. (1995) argued that, in natural clouds, small graupel after forming through dominant riming 205 growth can encounter predominant vapour growth when falling into such weak-LWC regions with LWC less than $0.1 \mathrm{~g} \mathrm{~m}^{-3}$. Even if such parts of any convective cloud are limited, the in-cloud motions must tend to mix the crystals throughout most subzero levels of the cloud over time. The inherent nonlinearity of ice multiplication occurring by such breakup, involving growth of splinters to become ice precipitation by a positive feedback (Yano and Phillips 2011), must make such active parts of the cloud disproportionately influential compared to their volume.

The contentious claim (sentence B) seems to rely on tacit additional criticisms of both lab/field experiments (Vardiman 1978; Takahashi et al. 1995) not included in the review (Korolev and Leisner, 2020, personal communication). We argue that these extra criticisms are mostly without merit as follows. First, there is the notion that somehow the weight of the fittings supporting the moving ice sphere in the Takahashi et al. (1995) experiment must have biased the measured fragment numbers. Indeed,

215 inspection of published diagrams of their apparatus reveals a third ice sphere on the opposite end of the metal nearly horizontal rotating bar (about $13 \mathrm{~cm}$ long, $3 \mathrm{~mm}$ wide) for the main moving rimed sphere (Takahashi et al. 1995), (Fig. 1). It has the same size as the other two spheres but does not collide when they do. We estimate that altogether the moment of inertia for the main rotating sphere ("A" in Fig. 1) about the central axis supporting the bar, was approximately doubled by including this extra sphere and the weight of the moving fixtures. Since the total initial CKE equals the rotational kinetic energy of the ice 220 sphere plus attachments (proportional to this moment of inertia) turning on the axis (almost vertical in Fig. 1), the initial CKE is roughly doubled by the extra mass. Hence, the extra weight for the rotating sphere acts to bias the measured numbers of fragments by only about 10\% in light of observations by Takahashi et al. (1995) at various impact speeds (Fig. 3). 
Equally, it is wrong to suggest that our formulation somehow did not consider rotational effects or that it is somehow ill-posed by not treating rotation explicitly. Rotational $\mathrm{KE}$ is included in Eq (1) underpinning our formulation and is only a small fraction of the total $\mathrm{KE}$ in any impact, as proven above.

Second, there is the criticism originally made by Phillips et al. (2017a) of the Vardiman (1978) experiment: ice particles were weakened by sublimation prior to collection outdoors, as they fell through the ice-subsaturated environment below cloud-base into the chamber on the mountainside where they broke up (Fig. 4). Phillips et al. reported that the prediction of $N$ from Eq (2), which for graupel-graupel collisions is fitted to only the Takahashi et al. (1995) observations, would be much lower (by about half an order of magnitude) than if Eq (2) were fitted only to observations by Vardiman (1978) for collisions of graupel, for the same size (the largest he observed). Yet in reality this was never a grave problem for our formulation because Phillips et al. (2017a) then applied a correction factor to the fragility coefficient, $C$, in Eq (2) to yield a match at this size between both predictions. Phillips et al. then applied this same correction factor to the formulation for other microphysical species (graupelsnow and graupel-crystal collisions) when constraining them with the Vardiman (1978) data alone. Prior sublimation artificially boosting the observed fragmentation of natural ice falling onto the mountainside was invoked as the reason for this empirical correction by Phillips et al.

Third, there is the notion that somehow the variation of rime density was not explored adequately in both lab/field experiments. Cloud-liquid properties and impact speed of cloud-droplets when accreted were variables not varied by Takahashi et al. (1995). However, the standard conditions for riming in the experiment by Takakashi et al. (see Corrigendum of KL2020) are representative of those in which large graupel are created in clouds. When the duration of vapour growth and riming of both ice spheres was varied from 5 to 15 mins, the number of fragments measured by Takahashi varied by a factor of about 2 .

To conclude, contrary to sentence B of the review, in view of such errors, the measurements of fragment numbers in ice-ice collisions by Vardiman (1978) and Takahashi et al. (1995) are not so defective as to render them useless for estimating coefficients in a theoretical formulation. Naturally, these coefficient values can easily be corrected as more accurate laboratory measurements become available in future, but without changing the formulation itself in any manner.

\subsection{Sublimational Breakup}

On the topic of sublimational breakup, KL2020 conclude that "this mechanism is also unlikely to explain explosive ice fragments may disappear by evaporation before they can be recirculated. Although that is indeed a major limitation, in 
reality during descent there must be continual emission of fragments and their continual depletion by total sublimation. This causes a dynamical quasi-equilibrium with an enhanced fragment concentration. So any mixing of downdraft air into the updraft will transfer air with the enhanced ice concentration into the updraft. Also, the entrainment of dry air and turbulence can cause a subsaturated environment, leading to the breakup, followed by possible mixing of fragments into convective ascent.

One can perform a scaling analysis: Oraltay and Hallett (1989) observed rates of emission of the order of $F \sim 0.1$ fragments per second per parent dendritic crystal (a few $\mathrm{mm}$ ) initially, during sublimation at relative humidities with respect to ice of about $70 \%$ or less. Such humidities would be attained in an adiabatic parcel descending at about $2 \mathrm{~m} / \mathrm{s}$ from about -15 degC

265 initially with about $n=3 \mathrm{~L}^{-1}$ of crystals initially $(2 \mathrm{~mm})$. If each fragment takes a minute $(\tau)$ to disappear by total sublimation, then the equilibrium number of fragments per parent particle is $F \tau \sim 0.1 \times 60=6$. Dong et al. (1994) observed rates of emission of $F \sim 0.3$ fragments per second per parent graupel particle (a few mm) initially. In a similarly subsaturated downdraft, there would be an equilibrium number of fragments per parent of $F \tau \sim 20$.

270 The pivotal point here is that such a quasi-equilibrium concentration is maintained throughout the entirety of the subsequent descent after being reached. Thus, any recirculation of downdraft air into the surrounding convective ascent would transfer air enriched in fragments for their subsequent vapour growth and survival.

\section{Conclusions}

The review of secondary ice production by Korolev and Leisner (2020, their Section 4) continues to depict our work (Yano and Phillips 2011; Yano et al. 2016; Phillips et al. 2017a) in a distorted manner, even after their Corrigendum. In particular, KL2020 made a contentious claim (sentence B above; Sec. 3) about the impossibility of developing any model formulation based only on the two existing experimental datasets of breakup in collisions of ice (Vardiman 1978; Takahashi et al. 1995). That claim is false and arose because Korolev and Leisner did not appreciate that our formulation of such breakup is based chiefly on an energistic theory coming from theoretical physics that is universally applicable to all collisions (Phillips et al. 2017a). The truth is that this formulation can be delivered even without quoting the results from any laboratory experiments. Korolev and Leisner then supposed that any unrepresentative aspect of the collisions in the experiment will somehow cause problems for calibrating the formulation, when in reality the formulation is based on such fundamental quantities that this is not a problem.

In reality, the two laboratory studies of fragmentation are not so erroneous as to prevent their use in calibrating theories of fragmentation in any ice-ice collision, such as ours. As noted above, our formulation does not necessarily require any such 
calibration. As far as we are aware, the only major issue with both lab/field experiments is a possible bias from sublimation of natural ice particles outdoors prior to their fragmentation observed by Vardiman (1978). But Phillips et al. (2017a) knew about this possible bias and corrected for it when calibrating their theory (Sec. 3.1). Anyway, the bias was not enough to alter the order of magnitude of $N$. It is a moot point whether the supposed sublimational weakening of these observations might actually have been representative of natural graupel aloft within clouds, since episodes of subsaturation with respect to ice are likely along the trajectory of any graupel particle while in-cloud.

More crucially, the errors in the breakup rate per collision from the formulation, quantified by Phillips et al. (2017a) as a factor of about 2 or 3, soon become immaterial in the context of explosive multiplication of ice concentrations in a natural cloud (Yano and Phillips 2011, 2016). For any ice multiplication somehow involving mixed-phase conditions (e.g. growth of fragments by riming to become graupel), the explosion occurs until an upper limit is reached (related to onset of water subsaturation), with little sensitivity to the breakup rate. Both published lab/field experiments we used are sufficient to allow a formulation that produces a simulation of observed cloud properties in agreement with aircraft observations of ice concentrations and many other related properties (Phillips et al. 2017b).

Finally, the possibility of sublimational breakup contributing to observed ice enhancement cannot be dismissed as easily as the review paper suggests. In reality, a dynamical quasi-equilibrium is established between emission and total sublimation of fragments, so that any region of sustained subsaturation with respect to ice will develop an enhanced ice concentration persisting throughout the descent. The enhanced ice can then be transferred into regions of ascent subsequently for growth. An order of magnitude of ice enhancement is possible from sublimational breakup in convective downdrafts and in their vicinity within the cloud. This quasi-equilibrium ice concentration was overlooked by KL2020.

In summary, the only two lab studies about breakup in ice-ice collisions hitherto (Vardiman 1978; Takahashi et al. 1995) are not so erroneous as to prevent their use in treating this process both in theoretical studies and numerical modeling of clouds, contrary to the claim by KL2020.

\section{References}

Dong, Y., R. G. Oraltay, and J. Hallett, 1994: Ice particle generation during evaporation. Atmos. Res., https://doi.org/10.1016/0169-8095(94)90050-7. 
320 Korolev, A., and T. Leisner, 2020: Review of experimental studies of secondary ice production. Atmos. Chem. Phys., 20, $11767-11797$

Oraltay, R. G., and J. Hallett, 1989: Evaporation and melting of ice crystals: A laboratory study. Atmos. Res., 24, 169-189

325 Phillips, V. T. J., Yano, J.-I., and A. Khain, 2017a: Ice multiplication by breakup in ice-ice collisions. Part I: Theoretical formulation. J. Atmos. Sci., 74, 1705-1719

Phillips, V. T. J., J. Yano, M. Formenton, E. Ilotoviz, V. Kanawade, I. Kudzotsa, J. Sun, A. Bansemer, A. G. Detwiler, A. Khain, and S. Tessendorf, 2017b: Ice multiplication by breakup in ice-ice collisions. Part II: Numerical Simulations. J. Atmos. Sci., 74, 2789-2811

Phillips, V. T. J., Patade, S., Gutierrez, J., and A. Bansemer, 2018: Secondary ice production by fragmentation of freezing of drops: formulation and theory. J. Atmos. Sci., 75, 3031-3070

335 Phillips, V. T. J., Formenton, M., Kanawade, V., Karlsson, L., Patade, S., Sun, J., Barthe, C., Pinty, J.-P., Detwiler, A., Lyu, W., Mansell, E. R., and S. Tessendorf, 2020: Multiple environmental influences on the lightning of cold-based continental convection. Part I: description and validation of model. J. Atmos. Sci., 77, 3999-4024

Supulver, K. D., F. G. Bridges, and D. N. C. Lin, 1995: The coefficient of restitution of ice particles in glancing collisions: Experimental results for unfrosted surfaces. Icarus, 113, 188-199

Takahashi, T., 1993: High ice crystal production in winter cumuli over the Japan Sea. Geophys. Res. Lett., 20, 451-454.

Takahashi, T., and K. Kuhara, 1993: Precipitation mechanisms of cumulonimbus clouds at Pohnpei, Micronesia. J. Meteor. Soc. Japan, 71, 21-31.

Takahashi, T., Nagao, Y., and Y. Kushiyama, 1995: Possible high ice particle production during graupel-graupel collisions. $J$. Atmos. Sci., 52, 4523-4527

350 Vardiman, L., 1978: The generation of secondary ice particles in clouds by crystal-crystal collision. J. Atmos. Sci., 35, 21682180

Vidaurre, G., and J. Hallett, 2009: Particle impact and breakup in aircraft measurement. J. Atmos. Oceanic Technol., 26, 972- 
https://doi.org/10.5194/acp-2021-123

Preprint. Discussion started: 15 February 2021

(C) Author(s) 2021. CC BY 4.0 License.

Wall, S., W. John, H. Wang, and S. L. Goren, 1990: Measurements of kinetic energy loss for particles impacting surfaces. Aerosol Sci. Technol., 12, 926-946

Yano, J.-I. and Phillips, V. T. J., 2011: Ice-Ice Collisions: An Ice Multiplication Process in Atmospheric Clouds, J. Atmos. Sci., 68, 322-333

Yano, J.-I., and V. T. J. Phillips, 2016: Explosive ice multiplication induced by multiplicative-noise fluctuation of mechanical break-up in ice-ice collisions. J. Atmos. Sci., 73, 4685-4697

365 Yano, J.-I., Phillips, V. T. J., and Kanawade, V., 2016: Explosive ice multiplication by mechanical break-up in ice-ice collisions: a dynamical system-based study, Q. J. Roy. Meteor. Soc., 142, 867-879 